\title{
Electron-Phonon Interaction in Electronic Tunneling: From Sequential Rate Equations to a Coherent Description
}

\author{
L.E.F. Foa Torres ${ }^{a}$, H.M. Pastawski ${ }^{a}$, and S. S. Makler ${ }^{b, c}$ \\ ${ }^{a}$ FaMAF, Universidad Nacional de Córdoba, Ciudad Universitaria, 5000 Córdoba, Argentina \\ ${ }^{b}$ Instituto de Física, Universidade do Estado do Rio de Janeiro, Rio de Janeiro, Brazil \\ ${ }^{c}$ Instituto de Física, Universidade Federal Fluminense, Campus da Praia Vermelha, 24210-340 Niterói, Brazil
}

Received on 23 April, 2001

\begin{abstract}
We discuss the main theoretical approaches for the phonon-assisted tunneling in double barrier resonant tunneling devices and introduce a quantum coherent treatment based on the mapping of the many-body problem into a higher dimensional one-body system. Conditions for a maximized phonon-emission are established.
\end{abstract}

Much progress in semiconducting and molecular electronic devices $[1,2]$ is inspired by Landauer's view $[3,4]$ of conductance as transmittance. However, the electron-electron (e-e) and the electron-phonon (e-ph) interactions add substantial complexity to the electronic problem, limiting its application. The first has received much attention in different contexts. In contrast, after the observation of optic phonon-assisted tunneling, [5] interest on e-ph interaction remained focused in double barrier Resonant Tunneling Devices (RTD). However, the recent observation of related electro-mechanical effects in molecular electronics [6] requires a reconsideration of the problem.

In a AlGaAs-GaAs RTD, besides the usual elastic resonance peak, a satellite peak rises in the valley of the current-voltage (I-V) curve. This occurs when the ground state in the well is one longitudinal optic (LO) phonon energy below the Fermi level of the emitter. Thus, an electron with kinetic energy $\varepsilon \leq \varepsilon_{F}$ and potential energy $e \mathrm{~V}$ in the emitter decays into an electron with energy $\varepsilon+e \mathrm{~V}-\hbar \omega_{0}$ in the collector plus a LOphonon. At this point, a number of questions emerge: Is it possible to extend the Landauer's picture to include inelastic scattering? What is the role of e-ph interaction in the electronic dephasing? In this work we discuss the main theoretical approaches used to treat the e-ph interaction in RTDs and try to shed light over these questions. Besides, the concept of resonance in e-ph Fock space is introduced.

The first solution of transport in a RTD including strongly inelastic e-ph scattering [7] considered a single electron state in the well interacting with optic phonons. The scattering problem was solved, in a one electron approximation, by computing the many-body Green's functions and resorting to some simplifications such as energy independent couplings to the electrodes [7] (broad band approximation). In this coherent picture, a tight-binding model [8] yields similar results.

A conceptually different approach, see Refs. [9] and [10], considered the e-ph interaction as a source of decoherence and thermalization for the electrons by adopting a complex self-energy correction to the electronic states. Thus, in this description, the phonon system acts in a way analogous to the "voltage probes" in the Büttiker's formulation of Landauer's picture. Only electrons that do not interact with phonons maintain coherence with its source. This line, which finds full formal support within the Keldysh formalism [11], has been further developed [12] to include strongly inelastic processes and originated computational codes [13] that simulate mesoscopic devices.

Most frequently rate equations $[14,12]$ are used. The calculation of the rate transition probabilities relies on the application of the Fermi Golden Rule (FGR) at two stages: a) To describe tunneling into the well. Quantum coherent effects are ignored since it is assumed that the phase of the electronic wave function is randomized by some mechanism. Then, within this sequential tunneling picture, the electron tunnels into the well and, after losing memory of its phase, it tunnels out of the well. b) To produce phonon emission. It requires a weak e-ph coupling with a dense phonon spectrum justifying the FGR and the electronic decoherence.

An alternative approach was introduced in Refs. [15] and [16]. There, the many-body problem of one electron interacting with phonons was exactly mapped into a one-body scattering system where each phonon mode adds a new dimension to the electronic variable. 
T o fix ideas, let us consider a simple Hamiltonian:

$$
\begin{aligned}
\mathcal{H}= & \sum_{j}\left\{E_{j} c_{j}^{+} c_{j}-V_{j, j+1}\left(c_{j}^{+} c_{j+1}+c_{j+1}^{+} c_{j}\right)\right\}+ \\
& +\hbar \omega_{0} b^{+} b-V_{g} \sum_{j \in \mathrm{w} \mathrm{ell}} c_{j}^{+} c_{j}\left(b^{+}+b\right),
\end{aligned}
$$

The first term represents a nearest-neighbor tight binding Hamiltonian for the electrons, where $c_{j}^{+}$and $c_{j}$ are electron operators at site $j$ on a $1-\mathrm{d}$ chain that includes a number of sites in the barriers and the w ell. The hopping parameters are $V_{j, j+1}=V$. The site energies $E_{j}$ model the potential profile. The second and third terms represent the phonon and the e-ph contributions. $b^{+}$and $b$ are the phonon operators and $V_{g}$ is the e-ph coupling that is limited to the w ell region. Then, if $\mathrm{w}$ econsider the Fock space expanded by $|j, n\rangle=c_{j}^{+}\left(b^{+}\right)^{n} / \sqrt{n !}|0\rangle$, the many-body problem maps to the 2-dimensional one-body problem shown in Fig. $1 \mathrm{a}$ ). The vertical dimension is the number $n$ of phonons $[15,16]$. The model can be further simplified by a decimation procedure if one considers only the electronic ground state in the well. Then, one gets a model for the RTD as a central site weakly coupled to the leads that interact with the phonons (see Fig 1 (b)). Then, $E_{0}$ is the well's ground state which is shifted by the electric field and $V_{0,1}=V_{\mathrm{R}}$ and $V_{-1,0}=V_{\mathrm{L}}\left(V_{\mathrm{L}(\mathrm{R})} \ll V\right)$ which fix the tunneling rates through the barriers.

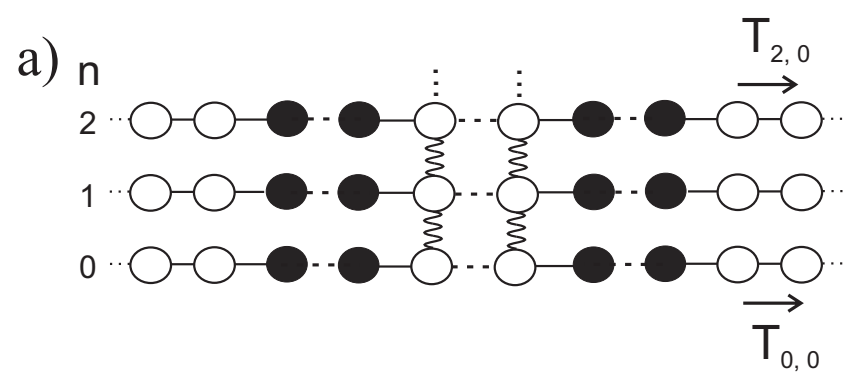

b)

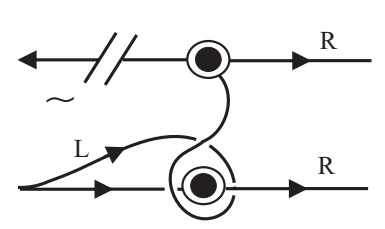

$\mathrm{L}$ c)

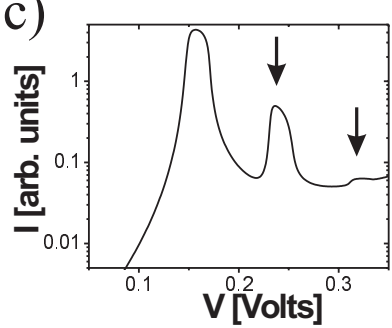

Figure 1. a) Each site is a state in the Fock space:The lo w er row are electronic states in different sites with no phonons in the well, the sites in black are in the barriers. Higher rows correspond to higher number of phonons. Straigh t lines are hoppings and w ary lines are e-ph couplings. b) Pictorial represen tation of the entangled processes of the first tw $o$ polaronic states. c) Calculated I-V curve showing satellite peaks of one and tw o phonon processes.

Within this equivalen t problem, the transmission probability of electrons between incoming and outgoing channels with different number $n$ of phonons can be calculated exactly from the Schr odinger equation. One can prune the Fock space and include only states within some range of $n$ allo wing a variational, non perturbative, calculation. Thus, we are not restricted to a w eak e-ph coupling. It must be emphasized that in this approach, no phase randomization caused of the e-ph in teraction is assumed. Instead of calculating transition rates, the complex quantum amplitudes for each state in the Fock space are obtained. T o calculate the transmittances between different channels sev eral methods can be adopted. One possibility is to solve for the wave function iterativ ely [16]. An alternative is to obtain Green's functions whose connection with the scattering matrix was established by Fisher and Lee and extended for multilead tight-binding systems by D'Amato and P astawski [10]. Here the pow er of the Green's functions techniques can be analytically exploited and transformed into computationally efficient algorithms. In this case, the horizontal dangling chains can be eliminated through a decimation procedure $[10,17]$ in troducing complex self-energies in the corresponding sites.

Once the transmittances are obtained, the question of ho w to compute the currents naturally emerge. In the Landauer's picture, the view is that of orthogonal scattering states extended along the conductor from the emitter to the collector. This orthogonality implies that the Pauli exclusion principle does not enter in the calculation of the currents. In the presence of inelastic scattering, electrons from different incoming states can occup y the same outgoing state.Thus, if one uses a single electron transmittances to represent the many-electrons system, these must be complemented with some factors accounting for the Pauli exclusion[18]. Otherwise, there may be an overflow of the final states. An attempt to solv ethis problem is the implementation of a selfconsistent procedure for the non-equilibrium electron distributions [19]. How ev erfor the exp erimental case of lo w temperatures and $\hbar \omega_{o}>\varepsilon_{F}$, there is no-overflow in the right lead since electrons with energies up to $\varepsilon_{F}$ cannot compete for the same final state. Then, the currents can be computed as in a multilead Landauer's picture. The total current from left to right is a sum of the currents through each of the leads on the right corresponding to different number of phonons:

$$
\mathrm{I}_{\mathrm{tot}}=\sum_{n} \mathrm{I}_{n}
$$

where, for high bias $\left(e \mathrm{~V}>\varepsilon_{F}\right)$,

$$
\mathrm{I}_{n}=\left(\frac{2 e}{h}\right) \int_{0}^{\varepsilon_{F}} T_{n, 0}(\varepsilon) \mathrm{d} \varepsilon
$$

$T_{n, 0}$ is the transmission probability from the left channel with no phonons to the channel with $n$ phonons in the collector.

Using controlled approximations, this approach allow edus to explore the resonances in the e-ph Fock 
space and to identify the control parameters in an RTD (device geometry, voltage) that optimize the coherent processes leading to the phonon emission. To illustrate this, we consider the states corresponding to 0 and 1 phonons. We found that the peak value of the inelastic transmission probability at the satellite peak is maximized when the in-scattering rate equals the out-scattering rate at the state with 1 phonon. The in-scattering rate $\tilde{\Gamma}_{\mathrm{L}}$ is equal to the rate of income to the state with no phonons, $\Gamma_{\mathrm{L}}$, reduced by a factor $\left(V_{g} / \hbar \omega_{o}\right)^{2}$ (see Fig. 1 b)). For $\left(V_{g} / \hbar \omega_{o}\right)^{2} \ll 1$ and $\Gamma_{\mathrm{L}}+\Gamma_{\mathrm{R}}<\hbar \omega_{o}$, the inelastic current is

$$
\begin{aligned}
\mathrm{I}_{1} & \simeq \frac{e}{\hbar} \frac{4 \widetilde{\Gamma}_{\mathrm{L}} \Gamma_{\mathrm{R}}}{\left(\widetilde{\Gamma}_{\mathrm{L}}+\Gamma_{\mathrm{R}}\right)} \times\left[\frac{2}{\pi} \arctan \left(\frac{\varepsilon_{F}}{2\left(\widetilde{\Gamma}_{\mathrm{L}}+\Gamma_{\mathrm{R}}\right)}\right)\right] \quad \\
& \simeq\left\{\begin{array}{cc}
\frac{e}{\hbar} 4 \widetilde{\Gamma}_{\mathrm{L}} \Gamma_{\mathrm{R}} /\left(\widetilde{\Gamma}_{\mathrm{L}}+\Gamma_{\mathrm{R}}\right) & \text { for } \varepsilon_{F} \gg\left(\widetilde{\Gamma}_{\mathrm{L}}+\Gamma_{\mathrm{R}}\right) \\
\frac{2 e}{h} T_{1,0} \times \varepsilon_{F} & \text { for } \varepsilon_{F} \ll\left(\widetilde{\Gamma}_{\mathrm{L}}+\Gamma_{\mathrm{R}}\right)
\end{array} .\right.
\end{aligned}
$$

Then, when $\varepsilon_{F} \gg\left(\widetilde{\Gamma}_{\mathrm{L}}+\Gamma_{\mathrm{R}}\right)$ the inelastic current becomes geometry independent in the wide range of $\varepsilon_{F} \gg \Gamma_{\mathrm{R}}>\widetilde{\Gamma}_{\mathrm{L}}$. In the opposite case $\mathrm{I}_{1}$, and hence the power emitted as phonons $\hbar \omega_{0} I_{1} / e$, becomes determined by the transmittance at resonance, which is maximized by the generalized symmetry condition $\widetilde{\Gamma}_{\mathrm{L}}=\Gamma_{\mathrm{R}}$. An $\mathrm{I}-\mathrm{V}$ curve maximizing phonon emission is shown in Fig. $1 \mathrm{c})$. This optimization can be useful for the generation of the primary longitudinal optic (LO) phonons in a SASER device $[15,20]$.

Finally, by noting that even if $\hbar \omega_{o} \rightarrow 0$ the outgoing currents in Eq. (2) can not interfere, we appreciate how the e- ph interaction introduces "decoherence" on the former single particle description. Within this formulation, decoherence arises because the inclusion of each phonon mode increases the "dimensionality" of the Hilbert space preventing the interference of the outgoing electron states.

\section{Acknowledgments}

We acknowledge financial support from CONICET, SeCyT-UNC, ANPCyT and an international grant from Andes-Vitae-Antorchas. HMP and LEFFT are affiliated with CONICET.

\section{References}

[1] L. P. Kouwenhoven et al., in Mesoscopic Electron Transport, L. L. Sohn, L. P. Kouwenhoven and G. Schön, Eds.
Kluwer 1997

[2] C. Joachim, J. K. Gimzewski and A. Aviram, Nature $408541(2000)$

[3] Y. Imry and R. Landauer, Rev. Mod. Phys. 71 S306 (1999)

[4] M. Büttiker, Phys. Rev. Lett.. 57, 1761 (1986)

[5] V. J. Goldman, D. C. Tsui, and J. E. Cunningham, Phys. Rev. B 36, 7635 (1987); M. L. Leadbeater et al., Phys. Rev. B 39, 3438 (1989); G. S. Boebinger et al., Phys. Rev. Lett. 65, 235 (1990).

[6] L. P. Kouwenhoven, Nature 40735 (2000); H. Park et al., Nature 407, 57 (2000); B. C. Stipe, M. A. Rezaei, and W. Ho, Phys. Rev. Lett. 81, 1263 (1998).

[7] N. S. Wingreen, K. W. Jacobsen, and J. W. Wilkins, Phys. Rev. Lett. 61, 1396 (1988).

[8] J. A. Støvneng, E. H. Hauge, P. Lipavský and V. Špička, Phys. Rev. B 44, 13595 (1991).

[9] S. Datta, Phys. Rev. B 40, 5830 (1989).

[10] J. L. D 'Amato and H. M. Pastawski, Anales AFA, 1, 239 (1989);Phys. Rev. B 41, 7411 (1990); see a tutorial description in H.M. Pastawski and E. Medina, Rev Mex. Fis. 47S1, 1 (2001) or cond-mat/0103219.

[11] H. M. Pastawski, Phys. Rev. B 46, 4053 (1992).

[12] R. G. Lake, G. Klimeck, M. P. Anantram and S. Datta, Phys. Rev. B 48, 15132 (1993).

[13] R. Lake, G. Klimeck, R. C. Bowen, and D. Jovanovic, J. Appl. Phys. 81, 7845 (1997).

[14] P. J. Turley and S. W. Teitsworth, Phys. Rev. B 44, 3199 (1991)

[15] E. V. Anda, S. S. Makler, H. M. Pastawski, and R. G. Barrera, Braz. J. Phys. 24, 330 (1994).

[16] J. Bonča and S. A. Trugman, Phys. Rev. Lett. 75, 2566 (1995); 79, 4874 (1997).

[17] P. Levstein, H. M. Pastawski, and J. L. D 'Amato, J. Phys. Condens. Matter 2, 1781 (1990).

[18] M. Wagner, Phys. Rev. Lett. 85, 174 (2000).

[19] E. G. Emberly and G. Kirczenow, Phys. Rev. B 61, $5740(2000)$.

[20] S. S. Makler, M. I. Vasilevskiy, E. V. Anda, D.E. Tuyarot, J. Weberszpil, and H. M. Pastawski, J. Phys. Condens. Matter 10, 5905 (1998); I. Camps and S. S. Makler, Solid State Commun. 116, 191 (2000). 